\title{
OPHTHALMIC CALCULATIONS BY THE "DAM" METHOD
}

BY

\author{
JOSEPH I. PASCAL \\ NEW YORK
}

IF one should ask for example the average ophthalmologist what is the power of a contact lens having given radii of say 8.5 and $8 \mathrm{~mm}$. and made of a given substance, say, $\mathrm{n}=1.488$ the chances are he would be stumped even though he had once learned the method of procedure. Most likely the same reaction would be encountered if he were asked to calculate the power of the cornea or of an ordinary ophthalmic lens.

There is a method for calculating surface power and lens power which is really very simple and once learned can never be forgotten. I have called it the "Dam" method, and I don't mean " damn." It is based on the formulation of a unit of curvature comparable to the unit of power which is the dioptre.

Everybody knows that a 1.00 dioptre lens has a focal length or briefly a focus of one metre or $100 \mathrm{~cm}$., or $1,000 \mathrm{~mm}$., or approximately 40 inches. It is sufficiently accurate to take one metre as equal to 40 inches. The relation between power in dioptres and focal length, or briefly focus, is expressed by these " key" numbers. To get power from focus or focus from power we divide into one of these key numbers. For example, what is the focus of a $4.00 \mathrm{D}$. lens ? Divide 4 into 100 , gives $25 \mathrm{~cm}$.; divide 4 into 1,000, gives $250 \mathrm{~mm}$., divide 4 into 40 gives 10 inches. Or conversely, what is the power of a lens having a focus of $20 \mathrm{~cm}$.? Divide 20 into 100 , gives us 5.00 dioptres. If the focal length is given in inches, say, the focus is 20 inches, divide 20 into 40 which gives $2.00 \mathrm{D}$. and so on.

Exactly the same relation that exists between power (in dioptres) and focus in metres, centimetres, millimetres, or inches, exists between curvature and radius in the same units. The radius just like the focal length may be expressed in metres, centimetres, millimetres, or inches. The curvature is expressed in a unit of curvature called a metrec (term derived from metrecurve). The relation between curvature (in metrecs) and radius is expressed by exactly the same key numbers as used in the relation between power (in dioptres) and focal length. Thus what is the curvature of a surface having a radius of $20 \mathrm{~cm}$. ? Divide 20 into 100 and we get 5 metrecs. Or what is the curvature of a surface having a radius of $8 \mathrm{~mm}$.? Divide 8 into 1,000 and we get 125 metrecs. Or what is the curvature of a surface having a radius of 10 inches? Divide 10 into 40 and we get 4 metrecs. 
The "Dam" formula is the general formula for surface power and is best written as $\mathrm{D}=\mathrm{aM}$. " $\mathrm{D}$ " stands for dioptres of power, "a" stands for the difference between the second index (that is the index of the medium where the light goes to) and the first index, i.e., the index of the medium where the light comes from. It can be written as " a " equals $\mathrm{N}_{2}-\mathrm{N}_{1}$. It may be memorised that " a" stands for the amount the second index is above the first index; $M$ stands for the curvature in metrecs. For example, what is the power of the anterior surface of the cornea having a radius of $8 \mathrm{~mm}$. and an index of 1.376? The radius and index must be given or assumed. Using our "Dam "

formula we get " a" $=1.376-1=0.376$, and $D=0.376 \times \frac{1000}{8}=\frac{376}{8}$

$=47 \mathrm{D}$. Or what is the power of the posterior surface of the cornea given a radius of $6.8 \mathrm{~mm}$. and the index of the aqueous as 1.336 ? Again $\mathrm{D}=\mathrm{aM}$, in which " $\mathrm{a}$ " is the difference between the second index, the aqueous humour on the other side of the surface, and the first index, which is that of the cornea itself (the posterior surface of the cornea separates the cornea from the aqueous humour). Here "a" equals $1 \cdot 336-1 \cdot 376=-0.040$. $\mathrm{D}=-0.040$ times $\frac{1000}{6 \cdot 8}=\frac{-40}{6.8}=-5.88 \mathrm{D}$.

The " $D=a M$ " formula gives the power of a surface and combination of surfaces in terms of reduced power. This is the power used by Gullstrand and other writers on physiological optics. For a surface bounded by air, or for a lens as ordinarily used, i.e., in air, the reduced power is the same as the actual power and we need not go any further into this.

In contact lens calculations since the radii are always given in millimetres the radius has to be divided into 1,000 . If one takes "a" to three decimal places and then moves the decimal 3 places, which multiplies it by 1,000 , one has only to divide this by the radius to get the power. Thus if a contact lens made of $n=1.488$ has radii of 8.7 and $8.5 \mathrm{~mm}$. the surface powers will 488 be $\frac{}{8.7}=+56.09$ for the anterior convex surface and $\frac{}{-8 .}=-57 \cdot 41$ 8.7 $-8.5$

for the posterior concave surface. If the lens is infinitely thin the total power is the sum of the surface powers, in this case $-1.32 \mathrm{D}$. In contact lenses the effect of thickness cannot be ignored and one must use a formula involving thickness.

This, however, is a secondary consideration and has to be used only after the surface powers are obtained. The "Dam" 
method after being used for a couple of examples gives a simple and easily retained method for all lens problems. Say what is the power of a thin ophthalmic biconvex lens of radii $25 \mathrm{~cm}$. and $40 \mathrm{~cm}$., made of glass index being 1.52 ? Using $\mathrm{D}=\mathrm{aM}$ for first surface, we get $\mathrm{D}=.52$ times $\frac{100}{25}=\frac{52}{25}=2.08 \mathrm{D}$. For posterior surface figuring that surface is convex to the outside, and the first index is air, we get $\mathrm{D}=52 \times \frac{100}{40}=\frac{52}{40}=1.30 \mathrm{D}$. Total power is $+3 \cdot 38 \mathrm{D}$.

The formula for the total power of a lens of any kind where the thickness has to be taken into account is not difficult to remember if one but thinks about it for a few minutes. The total or combined power is the sum of the surface powers with a modifying term. The formula consists of three terms, two of which are the surface powers and the third is the modifying term. Thus $\mathrm{Dp}=\mathrm{D}_{1}+\mathrm{D}_{2}-\mathrm{D}_{1} \mathrm{D}_{2} \mathrm{e}$. Dp stands for combined principal power, $D_{1}$ is the power of the first surface, $D_{2}$ is the power of the second surface. The sum of these two gives the basic power of the whole lens. The "modifying" third term is the product of these two powers, times the equivalent thickness "e." The equivalent thickness " $\mathrm{e}$ " is the actual thickness expressed in metres and divided by the index of refraction. Thus if the thickness is $3 \mathrm{~mm}$. and the index is $1.50, \mathrm{e}=\frac{0.003}{1.5}$, or if the thickness is $5 \mathrm{~mm}$. and the index 1.488 , " $\mathrm{e}$ " equals $\frac{0.0005}{1.488}$.

In short, the sum of the surface powers, minus their product times " $e$ " gives the total power. The formula is best remembered when read just as written, “ $D p$ equals D one plus D two minus D one D two e." In the contact lens example given above assuming the thickness to be $1 \mathrm{~mm}$. the modifying 001

third term is 56.09 times $-57.41 \times-$. This works out to 1.488

+216 . Adding $2 \cdot 16$ to the sum of the surface power -132 gives us a true principal power of $+0 \cdot 84 \mathrm{D}$.

The effect of thickness in this instance is very marked. In most contact lenses it is very much less marked. In ophthalmic lenses in general the effect of the thickness is negligible. So that if one forgets everything except the "Dam" method he will still be able to calculate all surface power, all thin lens power, and at least approximately nearly all "thick" lens problems. 